\title{
1 Assessing Tolerance to Heavy-Metal Stress in Arabidopsis \\ 2 thaliana Seedlings
}

3

4 Estelle Remy and Paula Duque

5 Instituto Gulbenkian de Ciência, Rua da Quinta Grande 6, 2780-156

6 Oeiras, Portugal

7

8

9

\begin{abstract}
Heavy-metal soil contamination is one of the major abiotic stress factors that, by negatively affecting plant growth and development, severely limit agricultural productivity worldwide. Plants have evolved various tolerance and detoxification strategies in order to cope with heavy-metal toxicity, while ensuring adequate supply of essential micronutrients at the whole-plant as well as cellular levels. Genetic studies in the model plant Arabidopsis thaliana have been instrumental in elucidating such mechanisms. The root assay constitutes a very powerful and simple method to assess heavy-metal stress tolerance in Arabidopsis seedlings. It allows the simultaneous determination of all the standard growth parameters affected by heavymetal stress (primary root elongation, lateral root development, shoot biomass and chlorophyll content) in a single experiment. Additionally, this protocol emphasizes the tips and tricks that become particularly useful when quantifying subtle alterations in tolerance to a given heavy-metal stress, when simultaneously pursuing a large number of plant lines, or when testing sensitivity to a wide range of heavy metals for a single line.
\end{abstract}


Key words Arabidopsis thaliana, Chlorophyll content, Heavy-metal stress tolerance, Lateral root development, Primary root elongation, Root assay, Seedlings, Shoot biomass

\section{Introduction}

As sessile organisms, terrestrial plants need to acquire their nutrients from the soil solution and therefore their growth and development largely rely on the soil mineral status. One of the most pervasive causes of loss of crop productivity worldwide is the contamination of arable land with heavy metals. Heavy-metal soil contamination as a result of anthropogenic activities occurs in many regions of the world and, depending on environmental as well as societal factors, may pose health risks to both humans and animals when accumulating in food crops. Given the modern agricultural context, the impact that this unfavorable soil condition exerts on crop yields will grow to paramount importance in the years to come. Thus, the elucidation of the physiological and molecular mechanisms underlying plant heavy-metal stress tolerance will be crucial for the use of biotechnology to reclaim farmlands lost to agriculture as well as in phytoremediation strategies - i.e., the use of plants to decontaminate polluted environments - and has been the subject of intense research in the plant biology field [1,2].

Heavy metals - i.e., in a broad sense, potentially toxic metallic elements such as zinc $(\mathrm{Zn})$, copper $(\mathrm{Cu})$ or iron $(\mathrm{Fe})$ among others, are essential for plant development as they serve as catalytic co-factors or structural motifs in numerous enzymes and other proteins assuming a key role in many basic metabolic processes. Nonetheless, these micronutrients become potentially toxic when present in excess. Conversely, non-essential heavy metals, such as cadmium (Cd), arsenate (As) or 
cesium (Cs) constitute toxic elements that can adversely affect plant growth even when present in trace amounts in the soil solution. Depending on the chemical and physical properties of the heavy metal in question, heavy-metal toxicity mainly occurs as a result of a propensity to inactivate crucial proteins through blocking of functional groups - the case of $\mathrm{Cd}$ and lead $(\mathrm{Pb})$ - or through displacement of essential metal

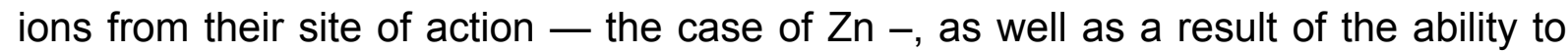
disturb cellular homeostasis of other essential elements - the case of As and Cs - or to induce oxidative stress through the generation of reactive oxygen species (ROS) the case of $\mathrm{Cu}$ and $\mathrm{Fe}[3]$.

To deal with these opposing effects and adjust to environmental fluctuations in their availability, plants have developed a sophisticated and tightly controlled homeostatic network aimed at ensuring an adequate supply of crucial oligo-elements while preventing the toxic build-up of both essential and non-essential heavy metals at the cellular and whole-plant levels [4]. Heavy metals are primarily acquired from the soil solution as ions, which once absorbed into the root epidermis move mostly symplastically through the adjacent cell layers to reach the central stele. After secretion into the stellar apoplast followed by active loading into the xylem vessels, heavy metals are translocated to the shoot via root pressure and the transpirational stream and subsequently transferred to the phloem sap before allocation to aerial organs. Plants adapt to essential heavy-metal shortage supply prevalently by activating cellular heavy metal uptake systems particularly at the root-soil interface [5]. At the whole-plant level, tolerance to heavy-metal excess is achieved mainly through reduced uptake at the root soil interface and through the rearrangement of its tissue partitioning via enhanced sequestration in leaves, whereas within the root both immobilization in the outer cell layers and exclusion from the epidermis contribute to 
limit heavy metal entry into the root symplasm. At the cellular level, such mechanisms are primarily intended to restrict the cytosolic accumulation of free heavy metal, mainly though extrusion in the apoplasm, chelation with specific ligands and/or vacuolar compartmentalization [6-8].

Deciphering the steps in plant heavy-metal stress tolerance and identifying the genetic determinants mediating heavy-metal uptake, translocation, chelation and detoxification largely benefit from molecular genetic studies in the plant model Arabidopsis thaliana. In the presence of excessive amounts of a given heavy metal, Arabidopsis seedlings develop pleiotropic toxicity symptoms, generally including shoot growth retardation, leaf chlorosis and remodeling of the root system architecture, i.e. inhibition of primary root elongation and altered lateral root development. The easiest and most commonly employed method to reliably appraise the extent of damage caused by heavy-metal stress in Arabidopsis seedlings remains the root assay $[9,10]$, which was initially adapted to heavy-metal susceptibility assessment by Howden and Cobbett in 1992 [11]. Root growth can be rapidly scored, and the assay requires relatively little specific equipment, providing both qualitative and quantitative data. It typically involves vertically-oriented growth of seedlings on solid media imposing or not a given rhizotoxic stress and scoring followed by comparison of primary root elongation in exposed versus non-exposed seedlings. Such an experiment can at first seem quite straightforward but often turns out to be rather challenging to interpret, in particular when a large number of lines of interest must be simultaneously compared, when a wide range of heavy metals has to be tested, or when subtle alterations between genotypes need to be reproducibly quantified. In addition, most of the reports using this assay focus merely on primary root elongation and disregard evaluation of other susceptibility indicators, such as 
shoot growth, photosynthesis performance or lateral root development. The present chapter does not aim solely at describing the root assay itself but rather to provide a precise practical application of the method, which is particularly helpful to accurately and fully evaluate Arabidopsis heavy-metal stress tolerance by measuring all standard phenotypic parameters in a single experiment. Using this combined approach, we were able to assign a role in ion rhizotoxicity tolerance to three Arabidopsis transporters from the Major Facilitator Superfamily. In particular, we reported that Pht1;9 function confers oversensitivity to As [12], while activity of the ZIF2 and ZIFL2 carriers promotes tolerance to $\mathrm{Zn}$ and $\mathrm{Cs}$ toxicity, respectively $[13,14]$.

\section{Materials}

\subsection{Plant Material}

1. Good-viability Arabidopsis seeds of the appropriate genotypes along with seeds of the corresponding wild type(s)

2. Seeds of previously reported tolerant and/or sensitive heavy-metal stress lines may also be used as controls (see Note 1)

\subsection{Reagents and Solutions}

1. MS (Murashige and Skoog, [15]) medium (or equivalent) freshly prepared before use: 1x MS basal salt mix, supplemented with $0.1 \mathrm{~g} \mathrm{~L}^{-1}$ myo-inositol and $0.5 \mathrm{~g} \mathrm{~L}^{-1}$ 2-(N-morpholino)ethanesulfonic acid (MES), $\mathrm{pH}$ adjusted to 5.7 with $\mathrm{KOH} 1 \mathrm{M}$, solidified with $0.8 \%$ ultrapur agar and autoclaved (see Note 2).

2. Sterilization solution freshly prepared before use: $50 \%[\mathrm{v} / \mathrm{v}]$ sodium hypochlorite and $0.02 \%[\mathrm{v} / \mathrm{v}]$ Triton $\mathrm{X}-100$ in sterile distilled water 
3. Appropriate antibiotics for seedling selection when mutant or transgenic seed batches are not derived from homozygous plants

4. Stock solutions of the heavy metals to be tested (Table 1)

\subsection{Consumables}

1. Eppendorf tubes (1.5 and $2 \mathrm{~mL}$ )

2. Fine forceps

3. Square Petri dishes $(11.5 \mathrm{~cm})$.

4. Razor blades.

5. Micropore tape $(3 \mathrm{M})$.

\subsection{Equipment}

1. A climate-controlled growth cabinet set to long-day (16-h light, $22^{\circ} \mathrm{C} / 8-\mathrm{h}$ dark, $\left.18^{\circ} \mathrm{C}\right)$ or under continuous light $\left(20-\mathrm{h}\right.$ light, $22^{\circ} \mathrm{C} / 4-\mathrm{h}$ dark, $\left.18^{\circ} \mathrm{C}\right)$ conditions with $60 \%$ relative humidity and cool-white light $\left(\sim 100-120 \mu \mathrm{mol} \mathrm{m}^{-2} \mathrm{~s}^{-1}\right)$

2. Space at $4^{\circ} \mathrm{C}$ (room or fridge)

3. Laminar flow chamber

4. Fume hood

\section{Methods}

The pipeline of the whole process is presented in Fig. 1.

1. Under sterile conditions, prepare square Petri dishes containing equivalent amounts of control MS medium or selective MS medium in case seed selection is required. Allow the plates to dry before closing them in order to avoid any condensation on the lid. 
2. Surface-sterilize the seeds by incubating them $10 \mathrm{~min}$ in sterilization solution (in Eppendorf tubes) under constant and vigorous shaking followed by four rinses with sterile distilled water.

3. Under sterile conditions, immediately spread evenly the seeds with the help of a tip onto the appropriate control plates (see Note 3 ) in 1-4 rows starting $1.5 \mathrm{~cm}$ from the top of the plate without spacing between the seeds (see Note 4). Allow the plates to dry until the water containing the seeds has totally evaporated. Seal the plates using Micropore tape and wrap them together in aluminum foil (see Note 5).

4. Incubate the plates vertically (see Note 6 ) at $4^{\circ} \mathrm{C}$ for 3 days to break seed dormancy.

5. After stratification, remove the aluminum foil and incubate the plates vertically (see Note 7) in the controlled-growth cabinet. Let the seeds germinate and the seedlings grow until root lengths reach roughly $1.0-1.2 \mathrm{~cm}$ (maximum $1.5 \mathrm{~cm}$ ). In our hands, this corresponds to about 4-6 days depending on the light conditions.

6. At this point, careful visual inspection of all plates is essential to ensure that the pre-defined experiment design is still feasible. First, it is crucial to ensure that the number of seedlings capable of being transferred is sufficient: seedlings of all the genotypes to be concomitantly tested must be at the same developmental stage, in particular with roots of similar length (less than $0.2 \mathrm{~cm}$ variation) and the plates must be free of fungal or bacterial contaminations. We typically transfer 16 seedlings per genotype per condition onto two different plates, each accommodating two genotypes in parallel, i.e. two sets of 8 seedlings. The appropriate controls should not be forgotten, i.e. transfer also of i) each genotype to a control plate to ensure that the phenotypic parameters to be measured are 
not altered under control conditions (and later normalize heavy-metal stress effects), and ii) seedlings from the wild-type background to each of the analyzed conditions. For studies where a single mutant or transgenic line is being compared to the corresponding wild type, the easiest way to ensure a valid comparison is to grow the wild-type seedlings on the same plate as the genotype under evaluation in order to avoid any effects of plate to plate variability. Alternatively, when a relatively high number of distinct genotypes in the same background need to be tested, 2-3 repetitions of wild-type seedling transfer in between the genotypes of interest may be acceptable to avoid extensive measurements.

7. Prepare square Petri dishes containing equivalent amounts of control MS medium and MS medium supplemented with the heavy metal(s) to be tested. Allow the plates to dry completely before closing them to avoid any condensation on the lid. Heavy-metal containing medium can be easily prepared by incorporating an appropriate amount of heavy-metal stock solution into previously autoclaved medium (see Note 8). The concentrations of the different heavy metals that we routinely test for the Arabidopsis ecotype Columbia (Col-0) are described in Table 1, but the appropriate concentrations should be empirically established depending on the accessions employed and the nature of the lines to be analyzed (tolerant or sensitive when compared to wild type). For a first screen, it is recommended to test a full range of heavy-metal concentrations.

8. Under sterile conditions, gently transfer seedlings using regularly disinfected forceps to the new plates by carefully allowing the root tip to touch the medium and, at an angle of approximately $30^{\circ}$, delicately sliding the root over the medium surface until the hypocotyl-root junction reaches a line drawn at $1.5 \mathrm{~cm}$ from the 
top of the plate. This way, the roots will be straight and contact with the medium surface will be maximized (see Note 9). Maintain a regular spacing between the seedlings. When the transfer is finalized, seal the plates, mark the position of the root tips directly on the bottom of the plate, and incubate plates vertically in the controlled-growth cabinet with roots pointing downward (see Note 10).

9. After approximately 7 days (see Note 11) of growth on new media, primary root elongation can be scored by marking the new position of the primary root tips (see Note 12).

10. After a further incubation period, i.e. just before the longest root of one genotype reaches the bottom of the plate, lateral root development can be recorded by first marking the new position of the primary root tips and then scanning the plates from their bottom side (see Note 13).

11. Immediately following scanning or after a further incubation period in the controlgrowth cabinet (see Note 11), seedlings can be assessed concomitantly for shoot biomass and chlorophyll content. Shoot biomass is determined by measuring the fresh weight of two pooled plant shoots (see Notes 13 and 14). Immediately after weighing, place the two plant shoots together in the bottom of a 2-mL Eppendorf tube and add $1 \mathrm{~mL}$ of $80 \%$ acetone. Once shoot biomass measurements are complete, incubate all the tubes overnight in the dark under gentle but continuous agitation. The following day, measure spectroscopically the absorbance of the acetone solution at $647 \mathrm{~nm}$ and $660 \mathrm{~nm}$.

12. Even before the end of the experiment, the primary root elongation and lateral root development parameters can be quantified for each seedling on scanned images using an image analysis software, such as ImageJ (http://rsb.info.nih.gov/ij/index.html). Primary root elongation is evaluated by 
measuring the exact distance between the initial and the corresponding mark. Lateral root density is evaluated by counting the number of lateral roots (excluding adventitious roots) and normalizing to the total length of the corresponding primary root, determined by measuring the exact distance between the hypocotyl-root junction and the final mark. Total lateral root length is evaluated by adding up the length of each lateral root of a given seedling. Total chlorophyll content is determined according to the method and equation (Total chlorophyll $\left.=18.71 A_{647 \mathrm{~nm}}+7.15 A_{660 \mathrm{~nm}}\right)$ of MacKinney [16] and expressed on a fresh weight basis (see Note 15). Finally, the average value of each of the

\section{Notes}

1. Best results will be produced if seed batches have been obtained from plants cultured simultaneously.

2. Before use, it is imperative that all soap, detergent and other cleaning fluids be completely removed from glassware, as even slight traces of such compounds in the medium will interfere with the assay. Glassware should be rinsed thoroughly 3 to 6 times with sterile distilled water before preparing the medium. Preparing the medium with sterile distilled water and avoiding storage of the plates before use significantly help preventing fungal and bacterial contaminations. 
3. The root assay can be carried out by germinating the seeds directly onto heavymetal containing plates or by transferring seedlings grown beforehand on control medium plates to heavy-metal containing plates. From our experience, the most informative is the transfer method described here, as it excludes a possible effect of heavy-metal stress on germination rate (which can be easily scored in an independent assay by measuring the germination rates of exposed versus nonexposed seedlings) while being relevant when seedling selection on medium supplemented with antibiotics is required prior to transfer.

4. Sowing the seeds at high density so that they touch each other on the plate rather than leaving space between them will greatly improve synchronization of seedling growth right after seed germination, in particular at the root level. In addition, this will favor straight growth of the roots and prevent their curling or curving. Another essential point when sowing the seeds is to avoid as much as possible scratching the medium surface with the tip to minimize root growth inside the medium rather than on its surface and thus prevent their subsequent transfer.

5. Spreading only one genotype per plate will prevent any cross-contamination between the lines to be tested. Alternatively, in the case of preliminary smallscale tests, two genotypes can be spread onto a single plate divided vertically. The number of seedlings amenable to transfer is often limiting, so be sure to plate enough seeds. The number of plates to be prepared depends on the germination rate of the seed batch and on the kind of screening to be performed (i.e., the type and range of heavy-metal concentrations to be tested). Nevertheless, we recommend preparing at least two plates per genotype in case fungal or bacterial contaminations appear. 
6. We have observed that performing the stratification step with Petri dishes set up already in a vertical position promotes synchronization of seedling root growth.

7. Petri dishes must be incubated vertically but with a slight inclination - i.e., a forward shift of $\sim 2 \mathrm{~cm}$ at the base of the dish - so that the seeds (and later the seedlings) face the light source. This will greatly favor the straight growth of the roots and is highly facilitated by the presence of gridded shelves (specific from the growth cabinet or, alternatively, from a fridge) holding the plates approximately at half their height. All the plates from a single experiment must be similarly inclined and if possible positioned on the same shelf so that they are exposed to the same amount of light.

8. Interpretation of the results can be erroneous if special care is not taken to ensure that the genotypes of interest are exposed to the exact same severity of heavy-metal stress as the wild-type control and thus uniform composition of the medium between plates is essential. We recommend preparing all the plates of a given concentration from the same heavy metal stock solution.

9. Transfer of the seedlings is the most critical step of the protocol. Its success depends largely on intact seedlings and any damage needs to be strictly avoided. Initially, it may take some practice of the transfer procedure to achieve quick transfer and correct positioning without wounding the seedlings, particularly squashing at the hypocotyl region. We strongly advise to delicately lift the seedling shoot using the fine forceps as a lever rather than closing them. If correct positioning is not achieved at a first attempt, make the seedling root slide again but never touch the root in order to preserve its integrity. To minimize dehydration of the seedlings, keep the lid of the initial and receiver plates as closed as possible during the transfer procedure. Any clearly wounded or dry 
seedling should be discarded. We highly recommend checking root integrity, in particular root tip intactness, of each transferred seedlings under a dissection microscope at the first transfer attempts, while routinely ensuring that 24 hours conditions). after transfer the roots have recovered and resumed steady-state growth even under heavy-metal stress (although at a slower rate than under control

10. Some studies indicate the inversion of the plates after transfer, so that the roots are pointed upward, to facilitate evaluation of primary root growth without having to mark root tip positions. However, we believe this method is only amenable to qualitative assessment of root elongation upon exposure to heavy-metal stress and largely privilege continuous growth as it allows the full extent of primary root elongation and lateral root parameters to be accurately measured, while eliminating possible effects of agravitropic behavior of the lines under evaluation.

11. One advantage of this method is that, as long as each specific trait is simultaneously quantified for all the genotypes under study, some slight variability in incubation times can be tolerated.

12. Susceptibility to heavy-metal stress will not necessarily follow a linear progression, particularly regarding primary root elongation. As scoring this parameter is a non-invasive method, we highly recommend marking the position of the root tips at 2-days intervals, at least in a first screen.

13. When recording phenotypical data, particular attention should be paid to the water frequently accumulating inside the plates. Water at the bottom of the plates can disturb seedling root position and it is crucial that they remain in place for later measurement from scanned images, while water condensed on the lid can easily wet seedling shoot and lead to highly erroneous conclusions. Keep the 
plates as vertical as possible before carefully opening the plate under sterile conditions, removing excess water by gently turning them over and drying the lid with paper. Seal back the plates in case further incubation is needed.

14. Still on the agar plates, cut two seedlings at the root-hypocotyl junction with a razor blade and immediately measure their combined weight using a precision weighing scale, while avoiding seedling damage as much as possible. Note that it is essential to be in a calm environment without frequent movements or strong ventilation to avoid quick water loss from the seedling shoots. For the same reason, keep the plates closed between each measurement.

15. Even taking particular care during plate preparation and seedling manipulation, contaminations frequently occur. Any contaminated seedling should be eliminated from the data recording, as should those that do not recover quickly after transfer or that suddenly arrest growth for no apparent reason. It is therefore important to follow the plates daily, as fungal and bacterial contaminations usually appear during prolonged incubation times. It should also be noted that shoot biomass and chlorophyll content can be assessed earlier than initially planned, i.e. as soon as a first plant shows signs of contamination, in order to save the experiment.

16. Be aware that the root assay is instrumental to determine the level of susceptibility to a given heavy metal, but not when the observed differences are due to altered internal heavy-metal homeostasis or whole-plant heavy metal accumulation. A similar assay to the one presented here, but set up on a larger scale, can be performed to prepare tissue samples for heavy-metal content quantification by methods such as atomic absorption spectroscopy. 


\section{Acknowledgments}

353

354

355

356

357

358

359

360

361

362

363

364

365

366

367

368

369

370

371

372

373

374

375

We thank Raquel Carvalho for critical reading of the manuscript. This work was supported by Fundação para a Ciência e a Tecnologia (Grant EXPL/AGRPRO/1013/2013 and post-doctoral fellowship SFRH/BPD/44640/2008 awarded to Estelle Remy). Funding of the research unit GREEN-it "Bioresources for Sustainability" (UID/Multi/04551/2013) is also gratefully acknowledged.

\section{References}

1. Hall JL (2002) Cellular mechanisms for heavy metal detoxification and tolerance. Journal of experimental botany $53(366): 1-11$

2. Rascio N, Navari-Izzo F (2011) Heavy metal hyperaccumulating plants: how and why do they do it? And what makes them so interesting? Plant science : an $\begin{array}{lllll}\text { international journal of experimental plant biology } 180 & (2): 169-181 .\end{array}$ doi:10.1016/j.plantsci.2010.08.016

3. Marschner P (ed) (2012) Mineral Nutrition of Higher Plants. Third Edition edn. Academic Press, London

4. Clemens S (2001) Molecular mechanisms of plant metal tolerance and homeostasis. Planta $212(4): 475-486$

5. Puig S, Penarrubia L (2009) Placing metal micronutrients in context: transport and distribution in plants. Current opinion in plant biology 12 (3):299-306. doi:10.1016/j.pbi.2009.04.008

6. Clemens S (2006) Toxic metal accumulation, responses to exposure and mechanisms of tolerance in plants. Biochimie 88 (11):1707-1719. doi:10.1016/j.biochi.2006.07.003 
7. Yadav SK (2010) Heavy metals toxicity in plants: An overview on the role of glutathione and phytochelatins in heavy metal stress tolerance of plants. South African Journal of Botany 76 (2):167-179. doi:10.1016/j.sajb.2009.10.007

8. Hossein MA, Piyatida P, Teixeira da Silva JA, Fujita M (2012) Molecular Mechanism of Heavy Metal Toxicity and Tolerance in Plants: Central Role of Glutathione in Detoxification of Reactive Oxygen Species and Methylglyoxal and in Heavy Metal Chelation. Journal of Botany 2012:Article ID 872875. doi:10.1155/2012/872875

9. Müller AJ (1964) Keimwurzeltest zur Bewertung des somatischen Strahlenschadens bei Arabidopsis. Die Kulturpflanze 12 (1):237-255

10. Contant RB (1966) Arabidopsis seedling growth for radiobiological studies. Arabid Inf Serv 3:36-37

11. Howden R, Cobbett CS (1992) Cadmium-Sensitive Mutants of Arabidopsis thaliana. Plant physiology 100 (1):100-107

12. Remy E, Cabrito TR, Batista RA, Teixeira MC, Sa-Correia I, Duque P (2012) The Pht1;9 and Pht1;8 transporters mediate inorganic phosphate acquisition by the Arabidopsis thaliana root during phosphorus starvation. The New phytologist 195 (2):356-371. doi:10.1111/j.1469-8137.2012.04167.x

13. Remy E, Cabrito TR, Batista RA, Hussein MA, Teixeira MC, Athanasiadis A, SaCorreia I, Duque P (2014) Intron retention in the 5'UTR of the novel ZIF2 transporter enhances translation to promote zinc tolerance in arabidopsis. PLoS genetics 10 (5):e1004375. doi:10.1371/journal.pgen.1004375

14. Remy E, Cabrito TR, Batista RA, Teixeira MC, Sa-Correia I, Duque P (2015) The major facilitator superfamily transporter ZIFL2 modulates cesium and potassium 
400 homeostasis in Arabidopsis. Plant \& cell physiology 56 (1):148-162.

401 doi:10.1093/pcp/pcu157

402 15. Murashige T, Skoog F (1962) A revised medium for rapid growth and bioassays 403 with tobacco tissue culture. Physiol Plant 15:473-497

404 16. MacKinney G (1941) Absorption of light by chlorophyll solutions. J Biol Chem $405 \quad 140: 315-322$

\section{Figure Legend}

408 Fig. 1. Overview of the protocol. Steps are referred to according to the Methods 409 section. Step 1, preparation of the plates; Step 2, surface-sterilization of the seeds;

410 Step 3, sowing of the seeds on control medium; Step 4, stratification of the seeds;

411 Step 5, germination and synchronized growth of the seedlings; Step 6, visual 412 inspection of the plates; Step 7, preparation of the heavy-metal stress and control 413 plates; Step 8, transfer and growth of the seedlings; Step 9, scoring of primary root 414 growth elongation; Step 10, scoring of lateral root development; Step 11, scoring of 415 shoot biomass and chlorophyll content; Step 12, analysis of the data. 
Table 1. Heavy-metal stock solutions and concentrations to test for the root assay in the Columbia (Col-0) ecotype of Arabidopsis thaliana.

\begin{tabular}{|c|c|c|c|c|c|}
\hline \multirow{2}{*}{$\begin{array}{l}\text { Heavy } \\
\text { metal }\end{array}$} & \multirow{2}{*}{ Cation } & \multirow{2}{*}{ Compound } & \multicolumn{2}{|c|}{ Stock solution } & \multirow[t]{2}{*}{ Range } \\
\hline & & & Concentration & Preparation & \\
\hline Aluminium & $\mathrm{Al}^{3+}$ & $\mathrm{AlCl}_{3}$ & $0.1 \mathrm{M}$ & $0.4 \mathrm{~g}$ in $30 \mathrm{ml} \mathrm{H}_{2} \mathrm{O}$ & $0.5,0.75,1,1.5,2 \mathrm{mM}$ \\
\hline Arsenate & $\mathrm{AsO}_{4}{ }^{3-}$ & $\mathrm{NaH}_{2} \mathrm{AsO}_{4}$ & $500 \mathrm{mM}$ & $0.82 \mathrm{~g}$ in $10 \mathrm{ml} \mathrm{H}_{2} \mathrm{O}$ & $100,200,300,400,500 \mu \mathrm{M}$ \\
\hline Cadmium & $\mathrm{Cd}^{2+}$ & $\mathrm{CdCl}_{2}$ & $30 \mathrm{mM}$ & $55 \mathrm{mg}$ in $10 \mathrm{ml} \mathrm{H}_{2} \mathrm{O}$ & $10,25,50,75,100 \mu \mathrm{M}$ \\
\hline Cobalt & $\mathrm{Co}^{2+}$ & $\mathrm{CoCl}_{2} \cdot 6 \mathrm{H}_{2} \mathrm{O}$ & $100 \mathrm{mM}$ & $0.238 \mathrm{~g}$ in $10 \mathrm{ml} \mathrm{H} \mathrm{H}_{2} \mathrm{O}$ & $25,50,75,100,150 \mu \mathrm{M}$ \\
\hline Copper & $\mathrm{Cu}^{2+}$ & $\mathrm{CuCl}_{2}$ & $30 \mathrm{mM}$ & $\begin{array}{l}51.1 \mathrm{mg} \text { in } 10 \mathrm{ml} \\
\mathrm{H}_{2} \mathrm{O}\end{array}$ & $25,50,75,100,150 \mu \mathrm{M}$ \\
\hline Iron & $\mathrm{Fe}^{2+}$ & $\mathrm{FeSO} 4$ & $0.1 \mathrm{M}$ & $0.278 \mathrm{~g}$ in $10 \mathrm{ml} \mathrm{H}_{2} \mathrm{O}$ & $0.25 \mathrm{mM}$ \\
\hline Lithium & $\mathrm{Li}^{2+}$ & $\mathrm{LiCl}_{2}$ & $5 \mathrm{M}$ & in $10 \mathrm{ml} \mathrm{H}_{2} \mathrm{O}$ & $5,10,12.5,15,20 \mathrm{mM}$ \\
\hline Manganese & $\mathrm{Mn}^{2+}$ & $\mathrm{MnCl}_{2} \cdot 4 \mathrm{H}_{2} \mathrm{O}$ & $0.5 \mathrm{M}$ & $0.990 \mathrm{~g}$ in $10 \mathrm{ml} \mathrm{H}_{2} \mathrm{O}$ & $1,1.5,2,2.5,3 \mathrm{mM}$ \\
\hline Nickel & $\mathrm{Ni}^{2+}$ & $\mathrm{Ni} \mathrm{Cl} 2.6 \mathrm{H}_{2} \mathrm{O}$ & $100 \mathrm{mM}$ & $0.238 \mathrm{~g}$ in $10 \mathrm{ml} \mathrm{H}_{2} \mathrm{O}$ & $50,75,100,150,200 \mu \mathrm{M}$ \\
\hline Lead & $\mathrm{Pb}^{2+}$ & $\mathrm{N}_{2} \mathrm{O}_{6} \mathrm{~Pb}$ & $0.5 \mathrm{M}$ & $\begin{array}{l}1.66 \mathrm{~g} \text { in } 9 \mathrm{ml} \mathrm{H}_{2} \mathrm{O}+ \\
1 \mathrm{ml} \mathrm{HNO}_{3}\end{array}$ & $0.1,0.25,0.5,0.75,1 \mathrm{mM}$ \\
\hline Cesium & $\mathrm{Cs}^{+}$ & $\mathrm{CsCl}$ & $5 \mathrm{M}$ & $8.42 \mathrm{~g}$ in $10 \mathrm{ml} \mathrm{H} \mathrm{H}_{2} \mathrm{O}$ & $1,2,3,4,5 \mathrm{mM}$ \\
\hline Thallium & $\mathrm{TI}^{3+}$ & $\mathrm{TICl}_{3}$ & $0.33 \mathrm{M}$ & $\begin{array}{l}3.1 \mathrm{~g} \text { in } 5 \mathrm{ml} \mathrm{HCl} \\
37 \%+25 \mathrm{ml} \mathrm{H}_{2} \mathrm{O}\end{array}$ & $2.5,5,10,15,20 \mu \mathrm{M}$ \\
\hline Zinc & $\mathrm{Zn}^{2+}$ & $\mathrm{ZnSO}_{4} .7 \mathrm{H}_{2} \mathrm{O}$ & $100 \mathrm{mM}$ & $0.288 \mathrm{~g}$ in $10 \mathrm{ml} \mathrm{H}_{2} \mathrm{O}$ & $100,250,500,750,1000 \mu \mathrm{M}$ \\
\hline
\end{tabular}

All heavy-metal stock solutions are prepared with sterile distilled water. 


\section{Figure 1}

STEPS 1 - 2 - 3

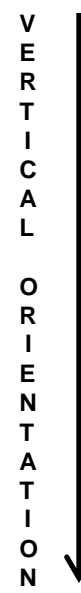

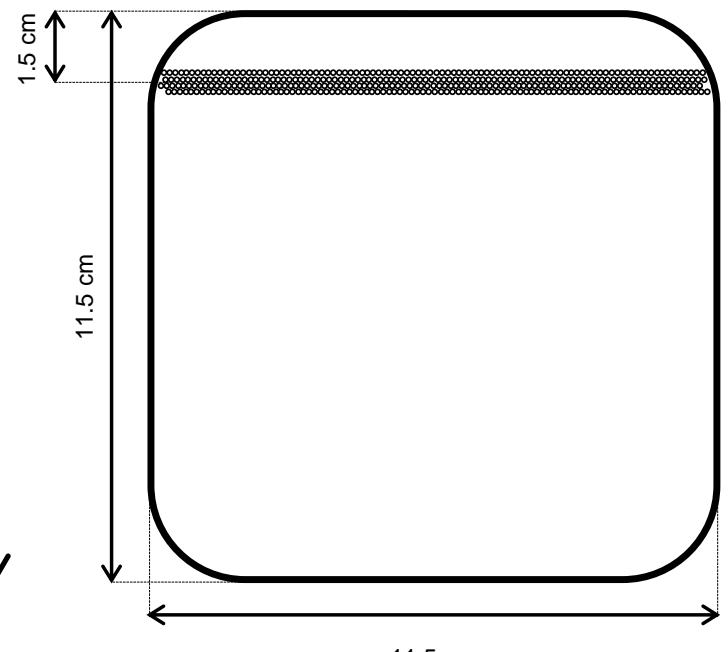

$11.5 \mathrm{~cm}$

\section{STEP 9}

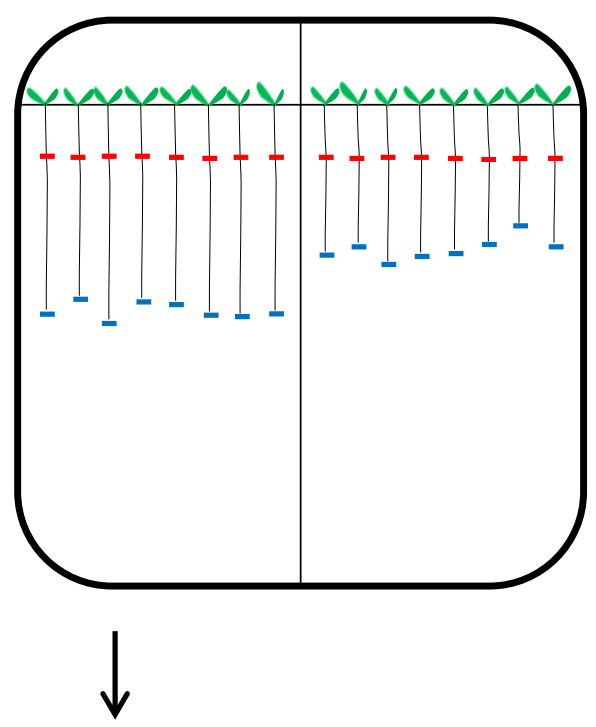

STEP 6

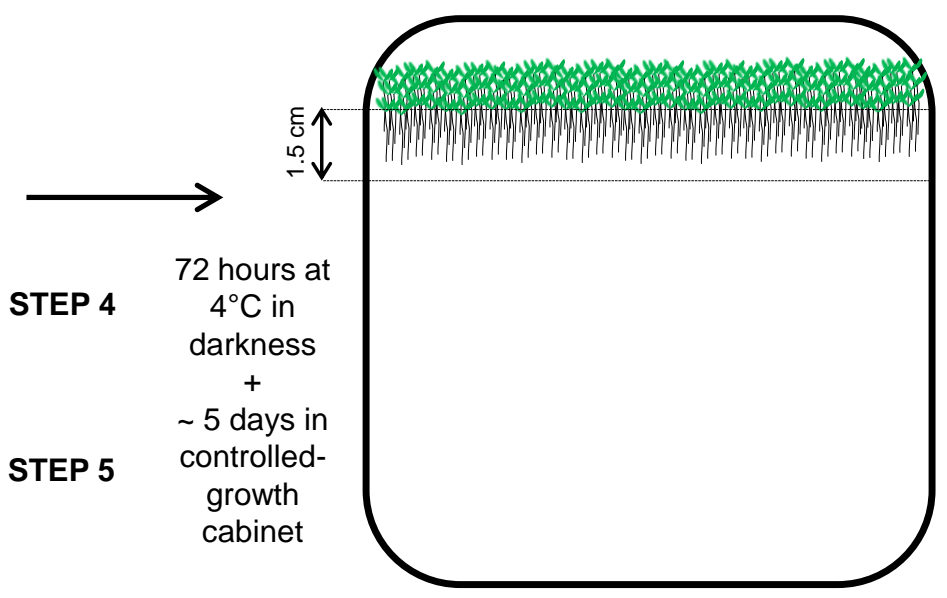

STEPS 7 - 8 $\begin{aligned} & \text { Transfer to new plates } \\ & \text { - Control medium } \\ & \text { - Heavy-metal stress }\end{aligned}$

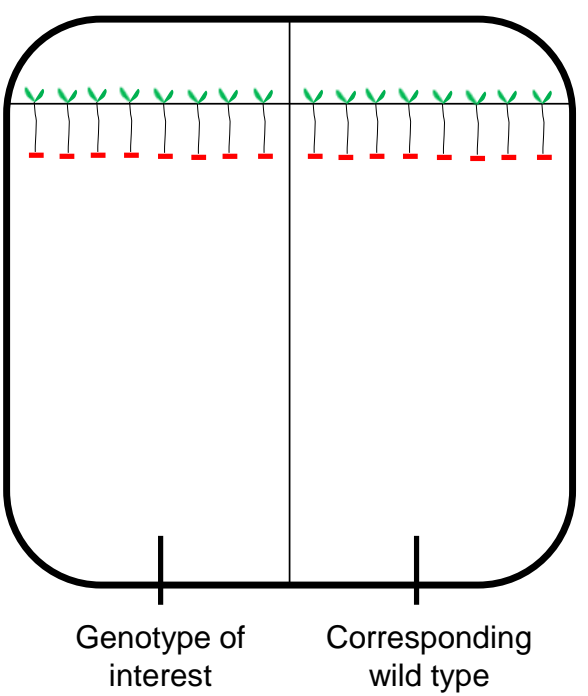

$\sim 7$ days in controlledgrowth cabinet under long-day conditions

\section{STEP 10}

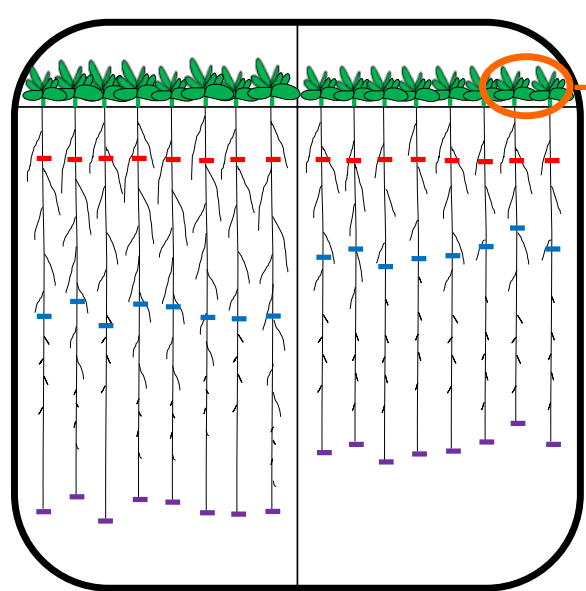

STEP 11

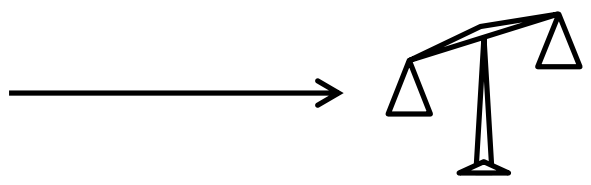

$\downarrow$

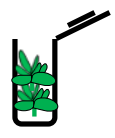

$+1 \mathrm{~mL}$ acetone $80 \%$
Overnight under agitation in the dark 